\title{
Factores asociados a depresión en médicos que trabajarán en zonas rurales del Perú.
} Factors associated with depression in doctors who will work in rural areas of Peru.

Christian R. Mejia ${ }^{1}$, Verónica Vega-Melgar ${ }^{1}$, Yanina Jiménez-Meza ${ }^{1}$, Jhosselyn I. Chacón ${ }^{2}$, Fiorella Inga-Berrospi ${ }^{1}$

Introduction: The doctors who perform the Peruvian service will be very exposed to loneliness, labor problems and others, which can have mental repercussions. The objective was to determine factors associated with depression among physicians who worked in rural Peru. Method: Observational, cross-sectional and analytical study of secondary data. Surveys were collected from newly graduated Peruvian doctors who conducted the Rural and Urban-Marginal Health Service in a few weeks. Depression was defined according to the Zung test, being associated to social and educational variables. Results: Of the 704 respondents, 30.0\% (211) had a score higher than 50 (depressed), 67.0\% (471) had a score close to the cut-off point for depression. In the multivariate analysis, it was associated with a higher frequency of female depression (RR: 1.09, 95\% CI: 1.09-1.10, $p$ value $<0.001$ ), having a sentimental partner (RPa: 1, 12, 95\% CI: 1.12-1.13, $p$ value $<0.001$ ), having studied at a university in Lima (RP: 1.07, 95\% CI: 1.06-1.09, $p$ value <0.001) And having medical relatives (RP: 1.20, 95\% CI: 1.09-1.33, p value <0.001); Adjusted by three variables. Conclusions: We found a significant percentage of physicians who had depression, another major group that was close to having it. It is important to carry out mental health programs among the doctors who perform the rural activity, since the conditions of their work generate problems in the styles of professional life.

Key words: Depression, rural health services, occupational health, physicians, Peru. (Source: DeCS BIREME).

Rev Chil Neuro-Psiquiat 2019; 57 (4): 320-328

Aceptado: 30/12/2019

Recibido: $23 / 04 / 2019$

Los Autores declaran no tener ningún conflicto de interés.

1. Escuela de Medicina Humana, Universidad Continental. Huancayo, Perú.

2. Federación Latinoamericana de Sociedades Científicas de Estudiantes de Medicina. Lima, Perú. 


\section{Introducción}

El Servicio Rural y Urbano-Marginal en Salud (SERUMS) es una actividad obligatoria para los profesionales de la salud que desean trabajar en instituciones públicas del Perú ${ }^{(1)}$. Es por ello que la gran mayoría de profesionales tienen que realizarlo, sobre todo porque si no lo realizan no podrán postular a la especialidad médica; esto genera una cierta obligación para realizar el trabajo en las zonas rurales del Perú (2). Por lo que anualmente son miles los profesionales de la salud que tendrán que realizar una migración laboral a zonas alejadas, y muchas veces agrestes, del territorio nacional ${ }^{(3)}$.

Han sido múltiples los reportes que han mencionado los problemas que se atraviesan en el SERUMS, que van desde los administrativos ${ }^{(4)}$, hasta los que se produjeron por diversas causas ${ }^{(5)}$. Todo esto genera un clima laboral singular, que muchas veces es la causa de que los profesionales se desanimen del trabajo en zonas de similares características ${ }^{(6)}$. Siendo importante el que se evalúe la salud mental de los que realizarán esta actividad, ya que, múltiples investigaciones locales muestran que los estudiantes del pre grado ya presentan problemas como Síndrome de Burnout ${ }^{(7)}$, o incluso depresión ${ }^{(8,9)}$. Es por todo eso que el objetivo del estudio fue el determinar los factores asociados a la depresión entre los médicos que trabajarán en zonas rurales del Perú.

\section{Metodología}

Se realizó un estudio observacional, de corte transversal y analítico, de un análisis de datos secundarios. Las encuestas fueron recolectadas entre los médicos peruanos recién graduados que realizarían el Servicio Rural y Urbano-Marginal en Salud (SERUMS) durante el período 2016-2017.

Se incluyó a los médicos que asistieron al curso de inducción al SERUMS, que ma- nifestaron que realizarían el SERUMS en el período 2016-2017 y que aceptaron participar en la investigación. Se excluyó a 23 encuestados por no haber respondido las preguntas del test de Zung, además, otras 50 encuestas fueron excluidas por no haber respondido las otras variables evaluadas (tasa de exclusión $=9,7 \%$ ).

Se definió depresión según el puntaje obtenido en el test de Zung, dicho test se compone de 20 preguntas cerradas de respuesta múltiple (que tiene cuatro posibles alternativas según la frecuencia de cada uno de los síntomas o estados que evalúa: poco tiempo, algo de tiempo, una buena parte del tiempo o la mayor parte del tiempo). Se consideró que tenían depresión positiva si tenían un puntaje igual o mayor de 50 (rango posible de puntaje: $20-80$ puntos).

Además, se midió otras características sociales y educativas de los encuestados: El sexo (masculino o femenino), la edad (medida en años), si actualmente tiene una pareja sentimental (si o no), la ubicación de su universidad (Lima o fuera de Lima), si ha estudiado alguna otra carrera antes de medicina (si o no) y si tenía familiares médicos (si o no).

Dicha encuesta se tomó en un evento que anualmente congrega a la mayor cantidad de médico que van a realizar su SERUMS, para su aplicación se coordinó para el pedido de permisos, la encuesta se realizó mientras los encuestados estaban en un intermedio de las exposiciones (cada uno en su asiento, sin distracciones y con las comodidades para la resolución de las encuestas). Luego de eso, dos de los autores realizaron el pasado de datos a doble digitación. Posteriormente se procedió a un proceso de depuración (según los criterios de selección) y control de calidad; este último fue realizado en dos pasos (uno previo realizado de forma manual por los autores estudiantiles, luego uno de los autores realizó el segundo control de calidad usando los filtros del programa Microsoft 
Excel). Toda esta información se exportó al programa estadístico Stata 11,1 (StataCorp LP, College Station, TX, USA).

Para el análisis estadístico se realizó un primer paso descriptivo, en donde se obtuvo las frecuencias y porcentajes de las variables categóricas, así como, las medianas y rangos intercuartílicos de las variables cuantitativas (post evaluación de los criterios de normalidad según la prueba estadística Shapiro Wilk). Luego se procedió a la realización de la estadística analítica, en donde se obtuvo las RP (razones de prevalencias), IC95\% (intervalos de confianza al 95\%) y valores p; todos ellos fueron obtenidos con los modelos lineales generalizados, con la familia Poisson, la función de enlace log, modelos robustos y usando el tipo de universidad como cluster de ajuste. Para la estadística analítica se tomó a los valores $\mathrm{p}<0,05$ como estadísticamente significativos.

La investigación respetó la ética en todo momento, los datos fueron confidenciales y solo manejados por el grupo que diseño el estudio. El proyecto de investigación base fue evaluado y aprobado por el comité de ética de la Universidad Peruana Cayetano Heredia (esto debido a que formaba parte de la medición basal de una tesis de maestría).

\section{Resultados}

De los 704 encuestados que cumplieron los criterios de selección, las mujeres tuvieron una mayor frecuencia de depresión ( $31,2 \%$ versus el $28,4 \%$ de los hombres), la edad no presentó diferencias según el tener depresión; además, también presentaron mayores frecuencias de depresión los que tenían pareja sentimental $(31,5 \%$ versus el $28,3 \%$ de los que no tenía pareja), los que estudiaron en una universidad de Lima Metropolitana (30,3\% versus el $27,6 \%$ que estudio en otras universidades), entre los que medicina humana fue su primera carrera $(30,2 \%$ versus el $27,3 \%$ de los que a tenían una carrera previa) y según el tener algún familiar médico $(32,8 \%$ versus el $27,3 \%$ de los que no tuvieron familiares médicos). Tabla 1.

Al obtener el puntaje del test de Zung, se encontró que el 30,0\% (211) tenía un puntaje mayor a 50 (que o clasificaba en el grupo de depresión); cabe resaltar que el 67,0\% (471) de los encuestados tuvo entre 41-49 puntos, que aún no los clasifica como deprimidos, pero que están en el área limítrofe de serlo. Figura 1.

En el análisis bivariado, se encontró que estaba asociado a la depresión el sexo $(p<0,001)$, el tener pareja sentimental $(\mathrm{p}<0,001)$, el estudiar en una universidad de Lima ( $p<0,001)$, el que medicina es su primera carrera $(p<0,001)$ y el que tenga familiares médicos $(\mathrm{p}<0,001)$. En el análisis multivariado, estuvo asociado a una mayor frecuencia de depresión el sexo femenino (RPa: 1,09; IC95\%: 1,09-1,10; valor $\mathrm{p}<0,001)$, el tener una pareja sentimental (RPa: 1,12; IC95\%: $1,12-1,13$; valor $p<0,001)$, el haber estudiado en una universidad de Lima (RPa: 1,07; IC95\%: 1,06-1,09; valor $\mathrm{p}<0,001)$ y el tener familiares médicos (RPa: 1,20; IC95\%: 1,09-1,33; valor $\mathrm{p}<0,001)$; estas variables estuvieron ajustadas por la edad, el que medicina humana fuese su primera carrera y el tipo de universidad. Tabla 2 .

\section{Discusión}

Se encontró que tres de cada diez médicos tenía depresión, y la gran mayoría tenía puntajes limítrofes para el diagnóstico de dicha patología. Esto es un hallazgo muy importante, ya que la ley peruana pide que las instituciones vigilen la salud de sus trabajadores, sobre todo las que son catalogadas en los rubros de alto riesgo -como es el caso del sector salud (10). Es por esto que las entidades contratantes, -el Ministerio de Salud, EsSalud, las fuerzas armadas y la sanidad de la policía- están en el deber de hacer exámenes ocupacionales que cuiden la salud mental de 
Tabla 1. Características sociales y educativas según el tener depresión entre los médicos que realizarán el servicio rural peruano.

\begin{tabular}{|c|c|c|c|}
\hline \multirow{2}{*}{ Variable } & \multicolumn{2}{|c|}{ Con depresión N (\%) } & \multirow{2}{*}{$\begin{array}{c}\text { Valor } \\
p\end{array}$} \\
\hline & SI & NO & \\
\hline \multicolumn{4}{|l|}{ Sexo } \\
\hline Femenino & $122(31,2)$ & $269(68,8)$ & 0,426 \\
\hline Masculino & $89(28,4)$ & $224(71,6)$ & \\
\hline Edad (años)* & $25(24-27)$ & $25(24-27)$ & 0,995 \\
\hline \multicolumn{4}{|l|}{ Tiene pareja } \\
\hline $\mathrm{Si}$ & $116(31,5)$ & $252(68,5)$ & 0,347 \\
\hline No & $95(28,3)$ & $241(71,7)$ & \\
\hline \multicolumn{4}{|l|}{ Estudio en } \\
\hline Universidad en Lima & $187(30,3)$ & $430(69,7)$ & 0,604 \\
\hline Otra Universidad & $24(27,6)$ & $63(72,4)$ & \\
\hline \multicolumn{4}{|l|}{ Es su primera carrera } \\
\hline $\mathrm{Si}$ & $196(30,2)$ & $453(69,8)$ & 0,649 \\
\hline No & $15(27,3)$ & $40(72,7)$ & \\
\hline \multicolumn{4}{|l|}{$\begin{array}{l}\text { Tiene familiares } \\
\text { Médicos }\end{array}$} \\
\hline $\mathrm{Si}$ & $113(32,8)$ & $232(67,2)$ & 0,114 \\
\hline No & $98(27,3)$ & $261(72,7)$ & \\
\hline
\end{tabular}

*Medianas (rangos intercuartílicos). Los valores p fueron obtenidos con chi cuadrado (variables categóricas) y la suma de rangos (variable edad).

Tabla 2. Análisis bivariado y multivariado de tener depresión en médicos que realizarán el servicio rural peruano según variables sociales y educativas

\begin{tabular}{lcccc}
\multirow{2}{*}{\multicolumn{1}{c}{ Variable }} & \multicolumn{2}{c}{ Bivariado } & \multicolumn{2}{c}{ Multivariado } \\
\cline { 2 - 5 } & RP $(\mathbf{I C 9 5 \% )}$ & Valor $\mathbf{p}$ & $\mathbf{R P a}(\mathbf{I C 9 5 \% )}$ & Valor $\mathbf{~}$ \\
\hline Sexo Femenino & $1,09(1,08-1,09)$ & $<0,001$ & $1,09(1,09-1,10)$ & $<0,001$ \\
\hline Edad (años) & $0,99(0,95-1,04)$ & 0,713 & $0,99(0,93-1,06)$ & 0,804 \\
\hline Tiene pareja & $1,13(1,08-1,18)$ & $<0,001$ & $1,12(1,12-1,13)$ & $<0,001$ \\
\hline Estudio en Lima & $1,08(1,04-1,12)$ & $<0,001$ & $1,07(1,06-1,09)$ & $<0,001$ \\
\hline Es su primera carrera & $1,05(1,05-1,06)$ & $<0,001$ & $1,04(0,77-1,39)$ & 0,808 \\
\hline Tiene familiares Médicos & $1,19(1,08-1,31)$ & $<0,001$ & $1,20(1,09-1,33)$ & $<0,001$
\end{tabular}

RP (razón de prevalencias), IC95\% (intervalo de confianza al 95\%), RPa (razón de prevalencias ajustado) y los valores $\mathrm{p}$ fueron obtenidos con los modelos lineales generalizados, con la familia Poisson, la función de enlace log, modelos robustos y usando el tipo de universidad como cluster de ajuste. 
Figura 1: Puntajes del test de Zung entre los médicos que realizarán el servicio rural peruano.

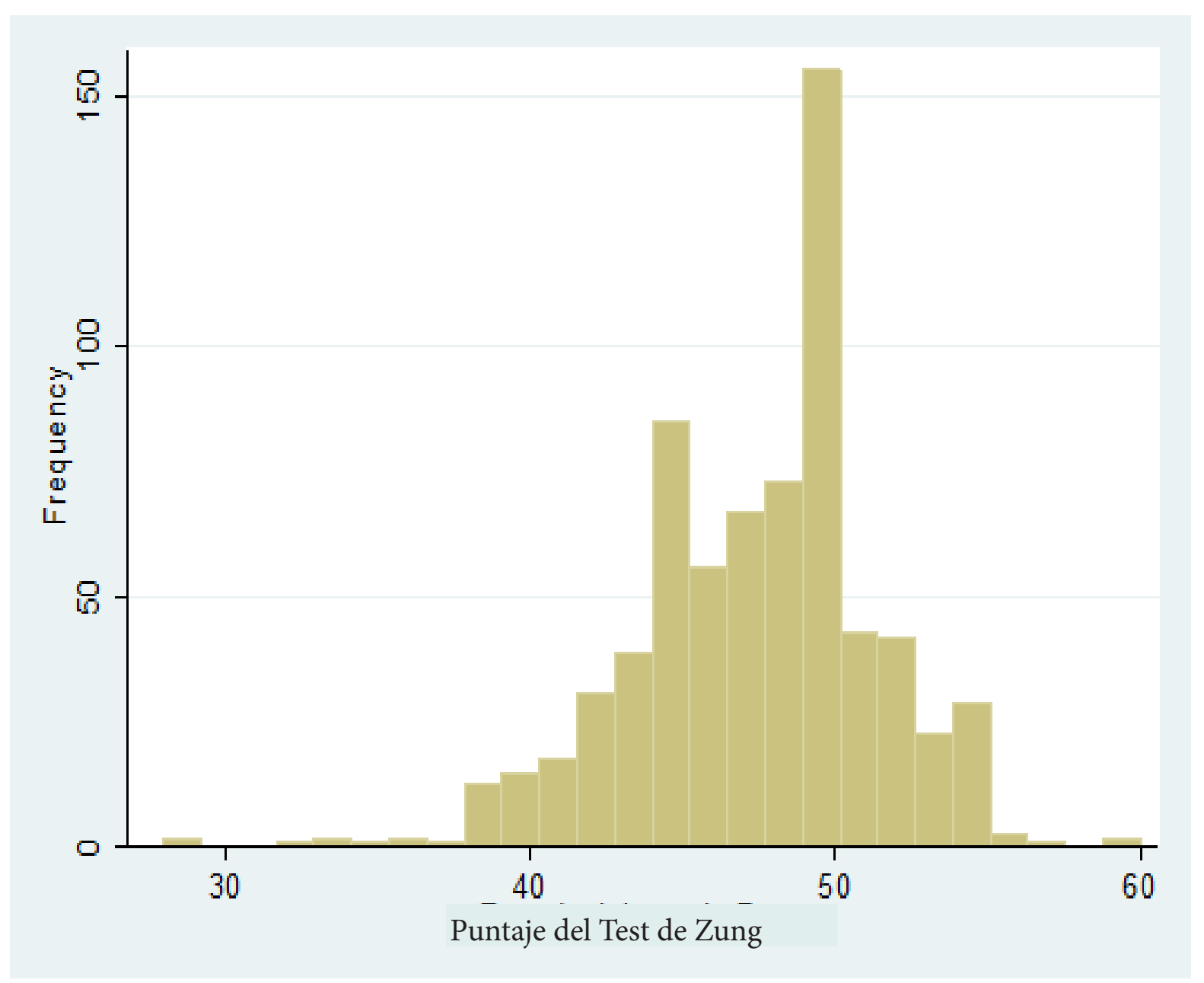

sus trabajadores, de lo contrario se exponen a las sanciones correspondientes ${ }^{(11)}$; esto debido a que atenta contra la salud mental de los trabajadores del sector salud. Estas sanciones tienen sus precedentes en realidades internacionales, en donde se cuida la salud y seguridad del trabajador, ya que si esto no se vigila se pueden producir diversos eventos adversos ${ }^{(12)}$. Sobre todo, debido a que esta patología ha sido reportado entre los estudiantes del pre grado en diversas facultades de medicina del Perú ${ }^{(9)}$, donde además de la depresión se ha reportado que pueden padecer de ansiedad, irritabilidad y otros trastornos crónicos ${ }^{(13)}$. Si esto no es atendido en la etapa del pre grado puede continuar hasta cuando ya son médicos ${ }^{(8)}$; por lo que se convierte en una patología importante en la práctica profesional de los médicos -y de otros profesionales de la salud-. Se recomienda que las instituciones encargadas realicen investigaciones que determinen el grado de afectación y las repercusiones que genera esta patología entre los médicos que realizan el SERUMS.

Se encontró que las mujeres tenían una mayor frecuencia de depresión que los hombres, esto es similar a lo reportado por Paula y Galán quienes han determinado que el ser mujer se asocia a un mayor riesgo de depresión ${ }^{(8,14)}$, llegando a significar incluso un factor de riesgo para desarrollar depresión 
crónica ${ }^{(15)}$, entre otras consecuencias que trae esta enfermedad. Probablemente esta condición se deba a aspectos sociales y fisiológicos de la mujer, que deberían ahondarse en sucesivos trabajos de investigación; para poder realizar programas específicos de detección y manejo de la misma.

Además, aquellos que tenían una pareja sentimental tuvieron mayor frecuencia de depresión, esto concuerda con los datos de una investigación que muestran que la mayor gravedad de síntoma depresivos se asocian a parejas disfuncionales ${ }^{(16)}$, pues la depresión está íntimamente relacionada con la calidad de la relación ${ }^{(17)}$. No se puede plantear causalidad (si el tener pareja sentimental causa depresión) debido al tipo de diseño que se usó (transversal), además, no se pudo tener más variables que caracterizaran el tipo de relación por ser un estudio de análisis secundarios de datos; por lo que se recomiendan que se realicen investigaciones de tipo longitudinal y con objetivos enfocados a conocer un poco más de esta temática.

Los egresados de universidades de la capital del país tuvieron más depresión; esto se podría atribuir a que el entorno en que se desempeña el estudiante en este caso es muy hostil, ya que en la capital existen diversos factores que podrían alterar la salud mental de las personas, como por ejemplo, el alto índice de delincuencia, corrupción, sensación de inseguridad ${ }^{(18)}$, contaminación ambiental y acústica, problemas derivados de la gran cantidad de población ${ }^{(19)}$, entre otros. No se encontró reportes similares en poblaciones parecidas a la evaluada, pero existe una publicación peruana que evaluó a pobladores rurales y reportó una menor frecuencia de depresión entre los pobladores de provincia y rurales ${ }^{(20)}$. Por lo que puede que estos profesionales puedan cambiar su nivel de depresión al exponerse a las sedes rurales donde realizarán su ejercicio profesional durante un año, por lo que son necesarias más investigaciones que puedan evaluar esto de forma prospectiva.

Por último, aquellos que tenían familiares médicos tuvieron más depresión, esto podría ser explicado porque reciben mayor presión por parte de sus familiares, lo que causaría un mayor nivel de estrés y esto precipitaría la sintomatología depresiva ${ }^{(21)}$.

Se tuvo la limitación del sesgo de selección, ya que el tipo de muestreo no permite realizar inferencia sobre la totalidad de médicos que realizarán el SERUMS, pero el objetivo de la investigación no fue el tratar de inferir a todos, sino el encontrar los factores asociados al padecer de esta enfermedad. Por otro lado, se tuvo el sesgo de información, ya que, al ser un análisis de datos secundarios no se pudo tener acceso a más variables importantes o a una mayor población, por lo que se recomienda que las instituciones relacionadas al tema planteen investigaciones que traten de abordar esta problemática.

Según lo encontrado, tres de cada diez médicos que trabajarán en zonas rurales padecen de depresión, estando asociada esta condición al sexo, el tener una pareja sentimental, el haber estudiado en una universidad de Lima y el tener familiares médicos. Se recomienda que las instituciones involucradas realicen mayor investigación en la temática, ya que estos profesionales se expondrán por todo un año a cosas positivas (la tranquilidad, bajos niveles de inseguridad, etc) y negativas (la soledad, lejanía de conocidos y actividades cotidianas, etc) del SERUMS, lo que puede traer diversas repercusiones en ellos. 


\begin{abstract}
Resumen
Introducción: Los médicos que realizarán el servicio rural peruano estarán muy expuestos a la soledad, problemas laborales y otros, que pueden tener repercusiones mentales. El objetivo fue determinar los factores asociados a la depresión entre los médicos que trabajarán en zonas rurales del Perú. Método: Estudio observacional, de corte transversal y analítico, de datos secundarios. Las encuestas se recolectaron entre los médicos peruanos recién graduados que realizarian en pocas semanas el Servicio Rural y Urbano-Marginal en Salud. Se definió depresión según el test de Zung, asociándose a variables sociales y educativas. Resultados: De los 704 encuestados, el 30,0\% (211) tenía un puntaje mayor a 50 (deprimido), el 67,0\% (471) tuvieron un puntaje cercano al punto de corte para depresión. En el análisis multivariado, estuvo asociado a una mayor frecuencia de depresión el sexo femenino (RPa: 1,09; IC95\%: 1,09-1,10; valor $p<0,001$ ), el tener una pareja sentimental (RPa: 1,12; IC95\%: 1,121,13; valor $p<0,001)$, el haber estudiado en una universidad de Lima (RPa: 1,07; IC95\%: 1,06-1,09; valor $p<0,001)$ y el tener familiares médicos (RPa: 1,20; IC95\%: 1,09-1,33; valor $p<0,001)$; ajustadas por tres variables. Conclusiones: Se encontró un importante porcentaje de médicos que tenían depresión, otro grupo mayor que estaba cerca de tenerlo. Es importante realizar programas de salud mental entre los médicos que realizarán actividad rural, ya que las condiciones de su trabajo podrían generar problemas en distintos aspectos de la vida profesional.
\end{abstract}

Palabras clave: Depresión, servicios rurales de salud, salud laboral, médicos, Perú. (fuente: DeCS BIREME).

\section{Referencias Bibliográficas}

1. Perú. Ministerio de Salud. Decreto Supremo 005-97-SA. Reglamento de la Ley 23330, Ley del Servicio Rural y Urbano Marginal de Salud - SERUMS. Lima: MINSA; 1997.

2. Mayta-Tristán P, Poterico JA, Galán-Rodas E, Raa-Ortiz D. El requisito obligatorio del servicio social en salud del Perú: discriminatorio e inconstitucional. Rev Peru Med Exp Salud Pública. 2014; 31(4):781-7.

3. Ministerio de Salud. Dirección General de Personal de Salud. Oferta de Plazas SERUMS [Internet]. [citado 5 de junio de 2017]. Disponible en: http://www.minsa.gob.pe/dggdrh/co- municate/antes.html

4. Mejia CR, Quiñones-Laveriano DM, Espinoza KG, Quezada-Osorio C. Deficiente cobertura de aseguramiento a médicos durante el servicio rural y urbano-marginal en Perú. Rev Peru Med Exp Salud Pública. 2014; 30(2): 220-23.

5. Mejia CR, Inga-Berrospi F, Albrecht Lezama C, García Q, Alejandra A. Problemas laborales en plazas del Servicio Rural Urbano-Marginal en Salud (SERUMS): auto-reporte de los médicos. Acta Médica Peru. 2016; 33(1):82-3.

6. Mejia CR, Quiñones-Laveriano DM. SERUMS y la migración de médicos: a propósito de una cohorte de médicos 
de Lima. Rev Peru Med Exp Salud Pública. 2015; 32(2):405-6.

7. Mejia CR, Valladares-Garrido MJ, Talledo-Ulfe L, Sánchez-Arteaga K, Rojas C, Arimuya JJ, et al. Síndrome de Burnout y factores asociados en estudiantes de medicina: Estudio multicéntrico en siete facultades de medicina peruanas. Rev Chil Neuro-Psiquiatr. 2016; 54(3):207-14.

8. Galán-Rodas E, Gálvez-Buccollini JA, Vega-Galdós F, Osada J, Guerrero-Padilla D, Vega-Dienstmaier J, et al. Salud Mental en médicos que realizan el servicio rural, urbano-marginal en salud en el Perú: Un estudio de línea base. Rev Peru Med Exp Salud Pública. 2011; 28(2):277-81.

9. Vargas M, Talledo-ulfe L, Samaniego RO, Heredia P, Rodríguez CAS, Mogollón CA, et al. Dispepsia funcional en estudiantes de ocho facultades de medicina peruanas. Influencia de los hábitos. Acta Gastroenterol Latinoam. 2016; 46(2):95-101.

10. Congreso de la República. Ley de Seguridad y Salud en el trabajo. Ley $N^{\circ}$ 29783. Diario Oficial El Peruano. 2012.

11. Mejia CR, Miraval-Cabrera E, Quiñones-Laveriano DM, Gomero-Cuadra R. Sanciones por infracciones contra la Salud y Seguridad en el trabajo en empresas de Perú, 2011-2013. Rev Asoc Esp Med Trab. 2015; 24(4):14957.

12. Men A. El papel del conocimiento experto en la gestión y percepción de los riesgos laborales. Arch Prev Riesgos Laborales. 2003; 6(4):158-65.

13. Mejia CR, Quezada-Osoria C, Verastegui-Díaz A, Cárdenas MM, García-Moreno KM, Quiñones-Laveriano DM. Factores psicosociales y hábitos asociados con dispepsia funcional en internos de un hospital nacional en
Piura, Perú. Rev Colomb Gatroenterologia. 2016; 31(4):354-9.

14. de Paula JA, Borges AMFS, Bezerra LRA, Parente HV, de Paula RCA, Wajnztejn R, et al. Prevalence and factors associated with depression in medical students. J Hum Growth Dev. 2014; 24(3):274-81.

15. Al-Faris EA, Irfan F, Van der VCP, Naeem N, Alsalem A, Alamiri N, et al. The prevalence and correlates of depressive symptoms from an Arabian setting: A wake up call. Med Teach. 2012; 34(s1):32-6.

16. Whisman MA, Baucom DH. Intimate Relationships and Psychopathology. Clin Child Fam Psychol Rev. 2012; 15(1):4-13.

17. Novak JR, Sandberg JG, Davis SY. The role of attachment behaviors in the link between relationship satisfaction and depression in clinical couples: implications for clinical practice. J Marital Fam Ther. 2016; 43(2): 1-12.

18. Diario La República. El Perú lidera lista de países con más víctimas de la delincuencia. Instituto de Estudios Peruanos [Internet]. Abril 2015. [citado 5 de junio de 2017] Disponible en: http://larepublica.pe/21-04-2015/elperu-lidera-lista-de-paises-con-masvictimas-de-la-delincuencia

19. Instituto Nacional de Salud Mental. Estudio epidemiológico de salud mental en Lima Metropolitana y Callao - replicación 2012. An Salud Ment. 2013; XXVIX(s1):269.

20. Quiñones-Laveriano DM, Espinoza-Chiong C, Scarsi-Mejia O, Rojas-Camayo J, Mejia CR. Altitud geográfica de residencia y dependencia alcohólica en pobladores peruanos. Rev Colomb Psiquiatr. 2016; 45(3):178-85.

21. Juseppe JER, Contreras RG, Mercado 
JMC. Prevalencia de Episodio Depresivo en los Médicos Residentes del Hospital Juárez de México. Rev Espec Méd-Quirúrgicas. 2005; 10(1):25-36.

Correspondencia:

Christian R. Mejia

Dirección: Av. Las Palmeras 5713 - Lima

39 - Perú.

Teléfono: 997643516

Correo: christian.mejia.md@gmail.com 